\title{
Dosimetric assessment of the impact of low-cost materials used in stereolithography in high-dose-rate brachytherapy
}

\author{
Grzegorz Bielęda, MSc'², Grzegorz Zwierzchowski, PhD',2, Katarzyna Rosłan, BSc³, Agnieszka Adamus, BSc³, \\ Prof. Julian Malicki, PhD',2 \\ 'Electroradiology Department, Poznan' University of Medical Sciences, Poznan̉, Poland, ${ }^{2}$ Medical Physics Department, Greater Poland Cancer \\ Centre, Poznan, Poland, 3Physics Faculty, Adam Mickiewicz University in Poznañ, Poznań, Poland
}

\begin{abstract}
Purpose: 3D printing has become a popular and widely available technique of rapid prototyping. The impact of used materials on the dose distribution has been studied for high energy sources. However, brachytherapy sources emit lower energy photons, and materials used in 3D printing may differ. This study was conducted to analyze the influence of common materials (polylactic acid - PLA and acrylonitrile butadiene styrene - ABS) used in stereolithography.

Material and methods: A 3D-printed phantom was designed, printed, and used to calibrate Gafchromic films. In the next step, a range of $1 \mathrm{~mm}$ thick plates of PLA and ABS (from zero to thirty) were inserted between source and detector to measure the impact of studied materials on delivered dose. Measurements were performed using a calibrated radiochromic film and Farmer ionization chamber in water.

Results: No statistically significant correlation $(p=0.4854)$ between the thickness of inserted PLA and the dose delivered to the film was obtained. With ionization chamber, Spearman's rank order test showed a significant correlation $(p=0.00004)$; however, the correlation was found weak. In case of ABS measurement, a statistically significant $(p=0.0159)$, yet weak negative correlation was found between the thickness of used material and the dose delivered to the film. On the other hand, a weak statistically significant $(p=0.0212)$ but positive correlation was found when the dose was measured with Farmer ionization chamber. We find these correlations false, as all measured doses were within the measurement uncertainty range (film $\pm 8.0 \%$, Farmer $\pm 8.8 \%$ ) from $100 \%$ of the prescribed dose.

Conclusions: According to obtained results, with the accuracy of measurement under clinical conditions, the impact of highly filled PLA and ABS printed objects on the dose distribution from an ${ }^{192}$ Ir source in water can be omitted.

J Contemp Brachytherapy 2021; 13, 2: 188-194 DOI: https://doi.org/10.5114/jcb.2021.105287
\end{abstract}

Key words: dosimetry, 3D printing, ABS, PLA.

\section{Purpose}

Stereolithography is one of the novel technologies that influences most aspects of modern life. 3D printing allows creating models for difficult surgeries, individual prosthesis, artificial limbs, and dose distribution modifications $[1,2]$. Moreover, this technique was introduced to radiation oncology and brachytherapy a few years ago. The potential to produce accessories needed for individual patients seems to be especially promising for clinicians and medical physicists. The possibility to adjust the actual dose distribution as close as possible to the ideal dose distribution has been the common goal of numerous researchers.
3D printing has been used in radiation oncology in order to prepare boluses to adapt doses to patient's shape in the treatment of tumors localized close to the skin. It applies especially in regions, which do not allow for the application of classical dose modifications. Individual patient immobilization accessories were introduced to limit patients' discomfort and to increase the repeatability of patients' position. Compensators, which allow to adjust whole radiation beams, are manufactured to adjust the field to meet the needs of specific patients.

Additive manufacturing has already been used in brachytherapy as well. Standard applicators are suitable for most situations but there are individual cases, which require a personalized approach. Individual applicators 
have been prepared for intracavitary gynecological, oral cavity, and skin surface treatment. Templates for needle insertion have been prepared to increase the precision of interstitial applications.

Fused deposition modelling (FDM) is one of the methods of rapid prototyping. In this technique, the material is formed as a filament wound on a spool and pressed through a nozzle heated to the material's melting point. The nozzle controls the flow of the material, and is moved automatically according to the instructions of the program. Various thermoplastic materials can be used in FDM, such as acrylonitrile butadiene styrene (ABS) and polylactic acid (PLA).

Introduction of new materials to prepare applicators for brachytherapy should be preceded by studies investigating the impact of these substances on radiation distribution. For low energy radiation used in brachytherapy, the photoelectric effect has a more significant contribution to the total radiation attenuation coefficient. The cross section $(\sigma)$ is proportional to the $4^{\text {th }}-5^{\text {th }}$ power of the atomic number $Z$, and is inversely proportional to the third power of radiation energy [3]. The attenuation coefficients for mono-energetic beams were defined only theoretically [4-6]. In case of wide energy radiation spectrum, this relation is of particular importance in conjunction with various attenuation coefficients of component radiation energies of the beam.

Based on the theoretically determined attenuation coefficients of iridium radiation source, first applicators applied in brachytherapy were produced using stereolithography $[7,8]$. First reports indicated that this technique is promising; however, it should be carefully verified prior to its common application.

The aim of the study was to determine the influence of ABS and PLA used in 3D printing on the dose distribution from ${ }^{192}$ Ir radioactive source.

\section{Material and methods}

\section{Polylactic acid}

Polylactic acid $\left(\mathrm{C}_{3} \mathrm{H}_{4} \mathrm{O}_{2}\right)_{n}$ is a thermoplastic and biodegradable aliphatic polyester obtained from naturally occurring organic lactic acid by condensation with loss of water. PLA is inexpensive, dimensionally stable, and it melts at a relatively low temperature (in the range of $180-220^{\circ} \mathrm{C}$ ), with glass transition temperature ranging from $60^{\circ} \mathrm{C}$ to $65^{\circ} \mathrm{C}$. It is most commonly produced for 3D printing as a filament with a diameter of $1.75 \mathrm{~mm}$. For this study, 30 plates $(100 \mathrm{~mm} \times 100 \mathrm{~mm} \times 1 \mathrm{~mm})$ were printed using a Makerbot Replicator plus printer. The printouts were performed in vertical orientation of plates, as there are technological problems with printing thin flat objects with FDM modality. Despite low susceptibility to deformation when cooling, the plates were slightly bent when printed horizontally, and more materials were wasted for providing a raft supporting the printout.

\section{Acrylonitrile butadiene styrene}

Acrylonitrile butadiene styrene $\left(\mathrm{C}_{8} \mathrm{H}_{8}\right)_{\mathrm{x}} \bullet\left(\mathrm{C}_{4} \mathrm{H}_{6}\right)_{\mathrm{y}}$ - $\left(\mathrm{C}_{3} \mathrm{H}_{3} \mathrm{~N}\right)_{\mathrm{z}}$ is a common thermoplastic polymer obtained by polymerization of styrene and acrylonitrile in the presence of polybutadiene. ABS is widely used for production of covers of electronic equipment, car elements, sport equipment, firearm components, decoration accessories, toys, and as a $3 \mathrm{D}$ printing material. It is inexpensive, it melts at a higher temperature ranging from $220^{\circ} \mathrm{C}$ to $260^{\circ} \mathrm{C}$, and its glass transition temperature is equal to approximately $105^{\circ} \mathrm{C}$. Due to higher melting temperature, it requires a heated worktable. In this study, 30 plates $(100 \mathrm{~mm} \times 100 \mathrm{~mm} \times 1 \mathrm{~mm})$ were printed using a Zortrax M200 printer.

\section{Phantom}

We have designed a $11 \mathrm{~cm} \times 11 \mathrm{~cm} \times 11 \mathrm{~cm}$ measurement phantom using a free online software Tinkercad (Figure 1A). This design allowed to place a square piece of film $(4 \mathrm{~cm} \times 4 \mathrm{~cm})$ in the center of cube (Figure 1B). The film holder was extendable from the phantom, and the whole construction was prepared using PLA. The phantom was designed to allow for insertion of a $30 \mathrm{~mm}$ thick layer of examined materials during second part of the experiment. A special insert for the Farmer chamber was designed and printed using PLA to ensure that the center of sensitive volume was aligned with the active dwell position (Figure 1C).
A

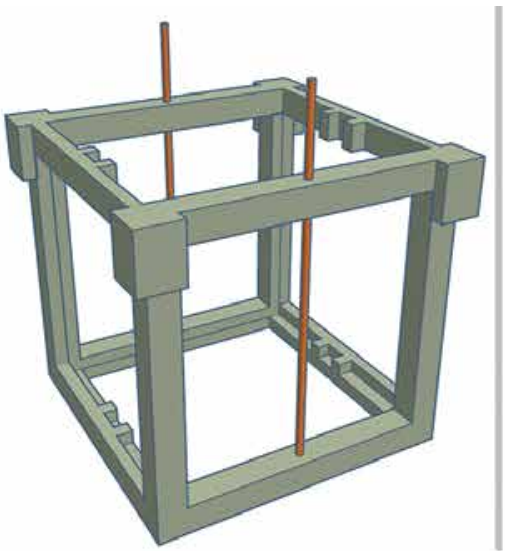

B

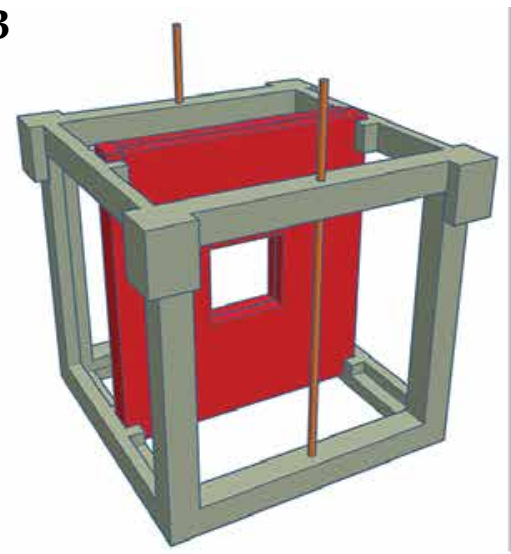

C

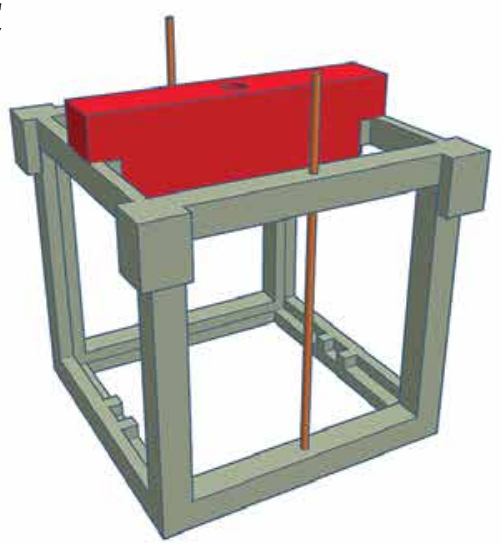

Fig. 1. Design of phantom used in presented study. A) Phantom with catheters for ${ }^{192}$ Ir source; B) Phantom with inserted film holder; C) Phantom with ionization chamber holder 


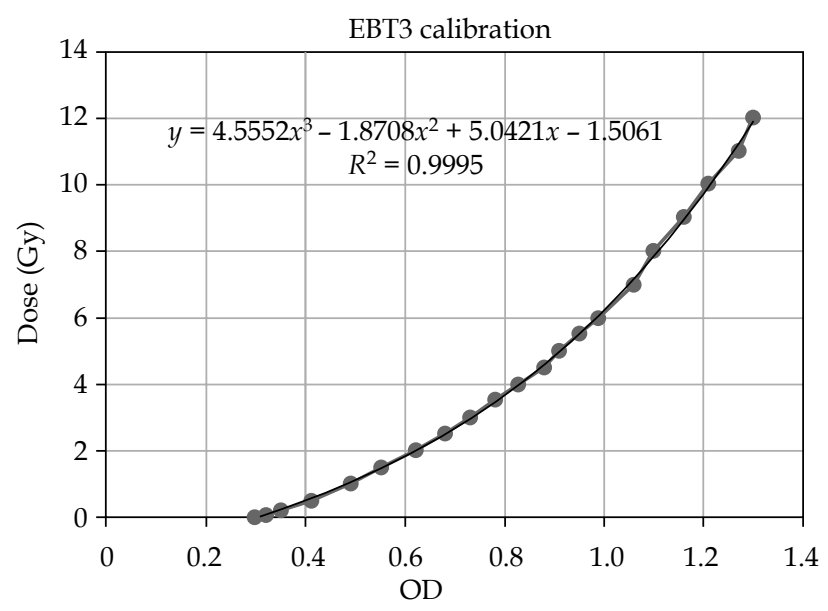

Fig. 2. Calibration curve for EBT3 Gafchromic film

\section{Film calibration}

In order to carry out proper measurement with radiochromic films, the sample from the used batch (lot No., 09131802) had to be calibrated. All the measurements were performed using microSelectron Digital (HDR-V3) brachytherapy afterloader (Nucletron, an Elekta company, Stockholm, Sweden) with ${ }^{192}$ Ir V3 source. The source was placed on both sides of the film, $5 \mathrm{~cm}$ from its center to reduce the heterogeneity of irradiated detector. Calculations carried out with Oncentra Brachy v.4.5.2 (Nucletron, an Elekta company, Stockholm, Sweden) showed that the reduction of dose heterogeneity due to bilateral irradiation was significant $(\mathrm{SD}=0.05 \%)$. Calibration was performed using a central $1 \mathrm{~cm} \times 1 \mathrm{~cm}$ square area. Dwell positions were defined using CT simulator lasers and a source position simulator used in clinical practice, to ensure that both the position of the source and the center of the film were lined up in plane perpendicular to the film. The films were irradiated using a water phantom in the same orientation with 20 points, ranging from $0.05 \mathrm{~Gy}$ to $12 \mathrm{~Gy}$, and scanned 72 hours after irradiation with Epson Perfection V750 Pro scanner.

\section{Dose measurement with radiochromic film}

The measurement was performed using the same phantom as film calibration. During this part of the experiment, the film was irradiated only from one dwell position in one catheter.

At the course of the experiment, 5 films were irradiated without any material between a source and a detector to check the repeatability of measurement setup. Afterwards, 30 measurements were taken, one after adding one-millimeter-thick plate of ABS and later PLA. This part of the experiment was repeated five times to reduce random errors. Consecutive series of measurements were performed one week apart. It was due to the accessibility of HDR (for a whole day experiment, HDR microSelectron was accessible only during weekends). Each film was irradiated with a $2 \mathrm{~Gy}$, as the dose readout was more stable with such a dose and the time needed to deliver it was acceptable. A microSelectron V3 HDR with ${ }^{192}$ Ir source (activity equal to $9.61 \mathrm{Ci}$ and delivery time of $415.6 \mathrm{~s}$ ) was used in the first attempt. The films were scanned in the same orientation 72 hours after irradiation. An area of 1 square centimeter was analyzed, as dose heterogeneity obtained from TPS was relatively low $\left(\mathrm{D}_{\min }=1.97 \mathrm{~Gy}\right.$, $\mathrm{D}_{\max }=2.02 \mathrm{~Gy}, \mathrm{SD}=0.011 \mathrm{~Gy}$ ).

\section{Dose measurement with ionization chamber}

The measurement was repeated using a calibrated PTW 30013 waterproof ionization chamber. The film holder was removed and replaced with an ionization chamber insert. The measurement was repeated using the same scheme as described for radiochromic film.

\section{Results \\ Calibration}

Overall, 22 film sheets were irradiated bilaterally in water with doses ranging from $0.05 \mathrm{~Gy}$ to $12 \mathrm{~Gy}$ to determine the calibration data. Seventy-two hours after exposition, they were scanned with 300 dpi resolution using a calibrated scanner. The central one square centimeter was analyzed to keep high-dose homogeneity. The calibration curve was determined as a third-degree polynomial:

$$
y=4.5552 x^{3}-1.8708 x^{2}+5.0421 x-1.5061
$$

Coefficient of determination of calculated polynomial was $R^{2}=0.9995$ (Figure 2).

\section{Polylactic acid}

A total of 31 sheets of EBT3 films were irradiated for measurement of PLA plates, starting with no PLA between the source and the detector, up to a $30 \mathrm{~mm}$ thick layer. After adding the next layer of tested material, the film was replaced. Each piece of the film was irradiated from one side with a 2 Gy dose. After 72 hours, the films were scanned with the same procedure as described for calibration films, and analysis was performed. The thickness of each tested material was measured five times. The central area of one square $\mathrm{cm}$ was analyzed, the doses measured are presented in Table 1. Spearman's rank order test showed no statistically significant correlation ( $p=0.4854)$ between the thickness of inserted PLA and the dose delivered to the detector, taking into account the assumed measurement error (Figure 3).

In the next step of the experiment, the film holder was replaced with the Farmer chamber mount, and a measurement with the ionization chamber was conducted. The dose of 2 Gy was deposited to the reference point on the chamber axis from one side. Unfortunately, it was difficult to stabilize the Farmer chamber, therefore setup uncertainty assumed for this part of the study was higher (Table 2). The data obtained during the measurement of PLA plates with the ionization chamber are presented in Table 1. Spearman's rank order test showed significant correlation ( $p=0.00004)$; however, according to Guilford classification, $r=-0.389164$ was a weak correlation.

\section{Acrylonitrile butadiene styrene}

The second series of measurements was performed to check the doses delivered to the film with ABS plates 
Table 1. Average values of measured dose and SD with corresponding thickness of studied materials inserted between source and detector. At the bottom $p$-value of Spearman's order rank test and $r$-value

Thickness

PLA

ABS

\begin{tabular}{|c|c|c|c|c|c|c|c|c|}
\hline & \multicolumn{2}{|c|}{ Film } & \multicolumn{2}{|c|}{ Farmer } & \multicolumn{2}{|c|}{ Film } & \multicolumn{2}{|c|}{ Farmer } \\
\hline & Average & SD & Average & SD & Average & SD & Average & SD \\
\hline 0 & 101.16 & 0.65 & 101.88 & 2.23 & 99.29 & 1.56 & 94.92 & 1.24 \\
\hline 1 & 101.18 & 1.71 & 100.40 & 2.53 & 102.09 & 1.70 & 96.40 & 1.17 \\
\hline 2 & 100.79 & 1.21 & 98.39 & 1.06 & 102.18 & 1.23 & 98.54 & 2.18 \\
\hline 3 & 99.94 & 1.84 & 100.13 & 2.08 & 101.89 & 2.03 & 95.97 & 1.48 \\
\hline 4 & 101.14 & 1.45 & 98.11 & 1.97 & 100.53 & 1.65 & 97.59 & 1.56 \\
\hline 5 & 101.21 & 1.46 & 101.08 & 2.45 & 99.81 & 0.72 & 98.97 & 1.32 \\
\hline 6 & 99.23 & 1.60 & 101.55 & 2.63 & 100.98 & 1.56 & 95.68 & 2.25 \\
\hline 7 & 101.59 & 0.65 & 102.04 & 2.49 & 100.84 & 1.62 & 95.44 & 2.89 \\
\hline 8 & 100.33 & 1.83 & 100.38 & 1.05 & 99.40 & 1.67 & 95.88 & 1.87 \\
\hline 9 & 100.90 & 1.46 & 98.94 & 2.34 & 99.78 & 1.61 & 94.98 & 2.26 \\
\hline 10 & 100.18 & 2.24 & 97.48 & 1.44 & 100.11 & 0.87 & 95.97 & 1.64 \\
\hline 11 & 99.67 & 1.99 & 99.26 & 2.10 & 102.10 & 1.01 & 97.87 & 0.47 \\
\hline 12 & 99.18 & 1.43 & 98.20 & 1.69 & 102.06 & 0.96 & 99.69 & 1.01 \\
\hline 13 & 98.85 & 1.17 & 100.83 & 3.43 & 99.86 & 0.55 & 96.39 & 2.06 \\
\hline 14 & 98.53 & 1.43 & 96.37 & 1.66 & 102.65 & 0.65 & 100.11 & 3.01 \\
\hline 15 & 98.69 & 1.11 & 100.96 & 2.12 & 101.57 & 1.14 & 99.70 & 2.23 \\
\hline 16 & 100.80 & 1.21 & 98.91 & 2.35 & 100.08 & 1.44 & 99.01 & 2.56 \\
\hline 17 & 99.24 & 1.67 & 99.50 & 2.01 & 99.44 & 1.63 & 99.48 & 0.80 \\
\hline 18 & 101.50 & 0.48 & 95.86 & 1.91 & 102.07 & 1.57 & 101.07 & 1.91 \\
\hline 19 & 99.72 & 1.18 & 100.18 & 2.79 & 99.84 & 0.80 & 99.13 & 2.18 \\
\hline 20 & 101.98 & 0.96 & 98.11 & 1.59 & 101.05 & 2.06 & 102.93 & 1.38 \\
\hline 21 & 100.56 & 0.96 & 99.14 & 1.93 & 99.62 & 1.51 & 101.26 & 2.81 \\
\hline 22 & 100.60 & 2.10 & 98.25 & 1.84 & 102.79 & 0.90 & 101.30 & 2.50 \\
\hline 23 & 100.20 & 0.81 & 97.95 & 1.85 & 100.72 & 1.78 & 102.45 & 0.89 \\
\hline 24 & 100.65 & 2.09 & 94.22 & 2.09 & 100.96 & 1.14 & 101.03 & 1.13 \\
\hline 25 & 101.45 & 1.62 & 98.16 & 1.42 & 98.79 & 1.23 & 102.75 & 2.01 \\
\hline 26 & 101.20 & 1.76 & 95.28 & 1.98 & 99.43 & 1.92 & 97.36 & 1.73 \\
\hline 27 & 99.49 & 1.29 & 97.65 & 1.78 & 98.63 & 0.90 & 99.37 & 2.12 \\
\hline 28 & 102.22 & 1.29 & 94.82 & 2.87 & 99.37 & 1.10 & 99.83 & 2.54 \\
\hline 29 & 100.33 & 1.61 & 94.09 & 1.94 & 98.62 & 1.28 & 101.53 & 1.68 \\
\hline 30 & 98.70 & 1.60 & 100.37 & 3.88 & 97.54 & 1.33 & 97.28 & 1.79 \\
\hline$p$ & \multicolumn{2}{|c|}{0.4857} & \multicolumn{2}{|c|}{0.00004} & \multicolumn{2}{|c|}{0.0159} & \multicolumn{2}{|c|}{0.0212} \\
\hline$r$ & \multicolumn{2}{|c|}{-0.061407} & \multicolumn{2}{|c|}{-0.389164} & \multicolumn{2}{|c|}{-0.216802} & \multicolumn{2}{|c|}{0.221230} \\
\hline
\end{tabular}

present in the experiment setup. The whole procedure was repeated similarly to PLA testing, and the data obtained from this part of the study are presented in Table 1. Although Spearman's test showed that a correlation may be established ( $p=0.0159)$, Guilford classification with $r=-0.216802$ classified it as weak.
The last part of the experiment was focused on the measurement of dose deposited to the Farmer chamber with variable thickness of ABS plates present between the source and the detector. Spearman's rank order test showed a significant correlation $(p=0.0212)$; however, according to Guilford classification, it was weak correlation $(r=0.221230)$. 

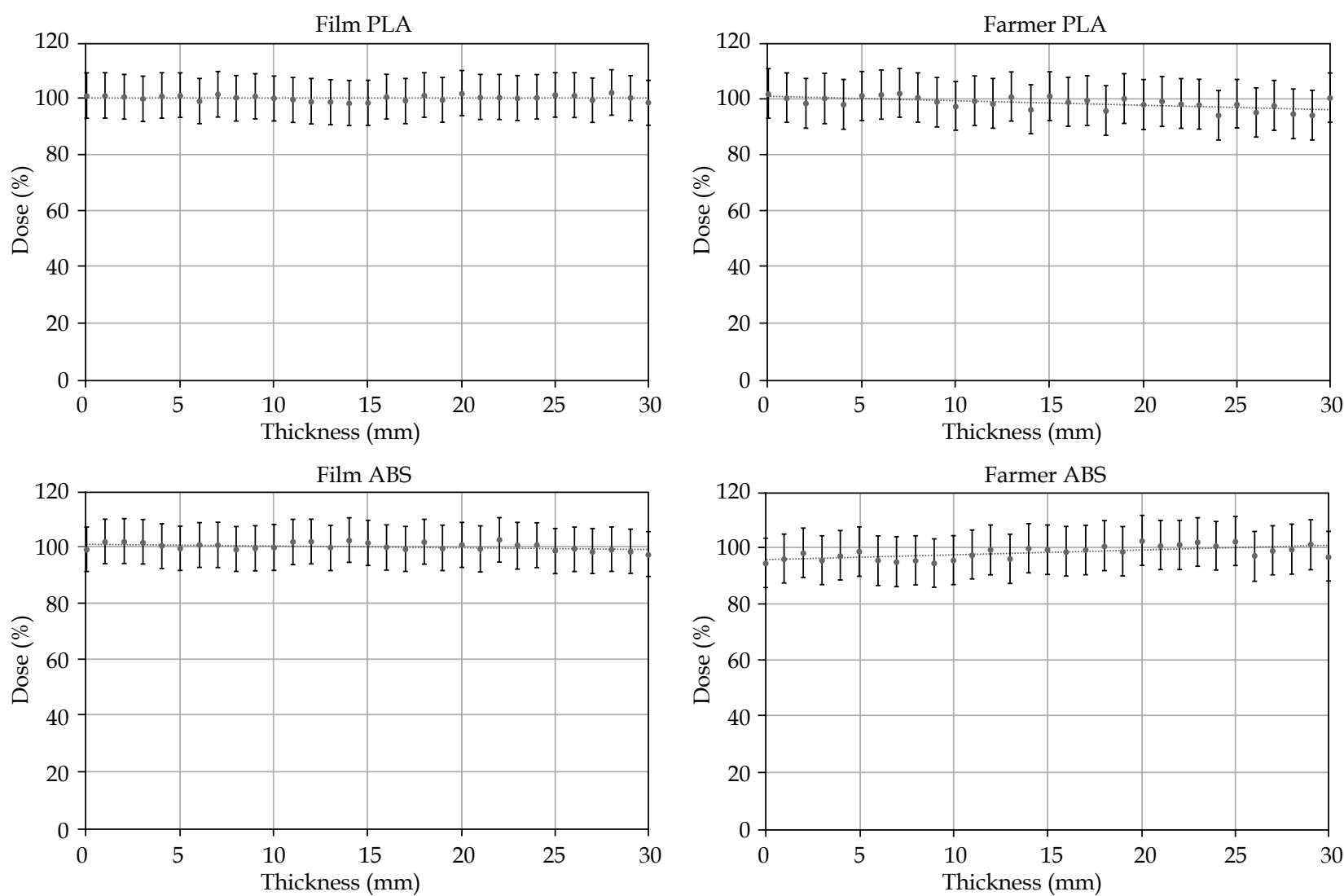

Fig. 3. Graphs presenting average dose and measurement uncertainty in correlation to thickness of studied materials. All measured doses are within measurement uncertainty from $100 \%$ of dose calculated in water within volume of interest

\section{Discussion}

Materials used in the FDM technique of 3D printing have already been tested regarding their impact on dose distribution in external beam radiation therapy, and were radiologically evaluated concerning the dependence between HU values and infill of prints [9-11]. In their study, Dancewicz et al. demonstrated that PLA and ABS printed structures with an infill of $90 \%$ are characterized by density similar to breast or adipose tissue when scanned with X-ray energies of $80 \mathrm{kVp}$ and $120 \mathrm{kVp}$. Their HU values were significantly higher when scanned with MVCT technique using a Tomotherapy Hi-Art unit, with an im-

Table 2. Measurement uncertainty analysis

\begin{tabular}{lcc} 
Variable & Film (\%) & Farmer (\%) \\
\hline Scanning consistency & 0.1 & 0.1 \\
\hline Film uniformity & 1.0 & 0.0 \\
\hline ROI size & 1.5 & 0.0 \\
\hline Calibration curve fit & 3.5 & 0.0 \\
\hline Chamber calibration & 0.0 & 3.0 \\
\hline Distance to source & 4.0 & 6.0 \\
\hline Source strength & 5.0 & 5.0 \\
\hline Exposure time & 0.5 & 0.5 \\
\hline Repeatability & 3.0 & 3.0 \\
\hline Total uncertainty & 8.0 & 8.8
\end{tabular}

aging beam energy of 3.5 MVp. These results ascertained us that the measurement with the iridium source is relevant. The maximum infill of prints used in this experiment was equal to $95 \%$; although given that the thickness of used materials sheets was equal to only $1 \mathrm{~mm}$ and the shell thickness was at $0.5 \mathrm{~mm}$, we assumed that the infill was between $95 \%$ and $100 \%$.

Additionally, theoretical calculations carried out using AutoZeff 1.7 software [12] showed that the PLA relative atomic number $Z$ is lower than water for radiation emitted by ${ }^{192} \mathrm{Ir}$ source, which implies lower dose attenuation than water, whereas that of ABS is slightly higher than water. Moreover, the mass density of PLA is $25 \%$ higher than that of water, and the ABS mass density is $6-8 \%$ higher than that of water. On the other hand, it is impossible to have $100 \%$ infill with FDM 3D printing technology, which may equilibrate the impact of different attenuation for iridium radiation spectrum [10].

In an experiment conducted by Sukhikh et al. [13], the characteristics of one batch of Gafchromic EBT3 polymer film for $10 \mathrm{MeV}$ and $2 \mathrm{MeV}$ electron beams, and $10 \mathrm{MV}$ and $70 \mathrm{kV}$ photon beams were investigated. The obtained relationships showed that the film response was the same in terms of uncertainty for electrons with energies of $2 \mathrm{MeV}$ and $10 \mathrm{MeV}$, but there was a significant difference for photons with energies of $70 \mathrm{kV}$ and $10 \mathrm{MV}$. On the other hand, Massillon et al. indicated that the EBT3 film is energy-independent at doses above $100 \mathrm{cGy}$ and at energies greater than $100 \mathrm{keV}$ photons, making it useful for 
${ }^{192}$ Ir sources with average photon energy of $380 \mathrm{keV}$ [14]. However, in the studies of Sukhikh et al., the dose was deposited with a linear accelerator, which facilitates calibration due to trouble-free positioning of the film at any distance from the radiation source as well as obtaining a homogeneous dose distribution in the entire fragment of deposited material.

In order to omit potential energy dependence of film calibration with different radiation source, we decided to perform film calibration with an iridium source, which was used in the following stages of our research. In this study, due to utilization of the ${ }^{192}$ Ir-point source, the irradiation of the film with a uniform dose was extremely difficult, therefore single dwell positions were activated in two opposite catheters to reduce dose heterogeneity in the irradiated area. This setup was built as a phantom printed with FDM technology using PLA material. Studies conducted by Orae suggest that use of PLA for calibration of EBT3 dosimetric film is justified [15]. On the other hand, we acknowledge that it may be reasonable to calibrate films using Linac setup due to high measurement uncertainty.

The second modality of measurement in this study was to apply the Farmer ionization chamber, as it is used for source strength checks in brachytherapy [16]. In our opinion, independent measurement obtained by employing a different technology consolidates the results, unfortunately it is burdened with even higher assumed measurement error.

The results of the performed measurements seem to be uncertain. Even with confirmed statistically significant correlations of Spearman's tests, they turned out to be weak according to Guilford classification. Furthermore, under clinical conditions, it is possible to perform measurements with correspondingly high measurement uncertainty. The AAPM Task Group No. 138 and GECESTRO estimated dose calculation uncertainty for high energy brachytherapy sources at a level of $6.8 \%$ [17]. Our estimation of uncertainty was slightly higher: $8 \%$ for the Gafchromic film and $8.8 \%$ for the Farmer chamber measurement. All the obtained results were within a measurement uncertainty range from $100 \%$ of the prescribed dose, regardless of the employed measurement method, type of used material, or its thickness. We consider statistically proven correlations to be confusing, since the correlation for ABS inserts measured with the film was negative $(r=-0.216802)$, whereas that measured with the ionization chamber was positive $(r=0.221230)$. In the case of PLA, there was no correlation for a film measurement and a statistically significant but weak correlation for the Farmer chamber measurement $(r=-0.389164)$.

Considering all stated above, we think that dose attenuation similar to water suggests that dose distribution calculated based on TG43 formalism is reliable. Different materials, which may be used for manufacturing of applicators or complementary accessories for brachytherapy can contribute to higher differences in terms of dose distribution. This effect can be omitted with dose calculation using model-based dose calculation algorithms; however, the majority of brachytherapy departments rely on a well-established TG43 estimation.
Furthermore, considering that potential applicators printed using ABS or PLA would position the source between 3 and $10 \mathrm{~mm}$ from the irradiated surface, the impact could be very low. Also, with high infill (over 90\%) of the printed structure, air gaps might have a much lower impact on dose distribution compared to air gaps in flap applicators supplied by standard applicator vendors.

\section{Conclusions}

Based on the obtained results and considering the accuracy of measurement under clinical conditions, it can be determined that the impact of highly filled PLA and ABS printed objects on the dose distribution from the Ir-192 source in water can be omitted. The calibration of radiochromic films with the iridium source is possible for brachytherapy; however, measurement uncertainty should be taken into account.

\section{Acknowledgements}

This study was conducted with a grant funding No. 502-14-04412527-11083 awarded by the Poznań University of Medical Sciences.

\section{Disclosure}

The authors report no conflict of interest.

\section{References}

1. Rankin TM, Giovinco NA, Cucher DJ et al. Three-dimensional printing surgical instruments: are we there yet? J Surg Res 2014; 189: 193-197.

2. Kadoya N, Miyasaka Y, Nakajima Y et al. Evaluation of deformable image registration between external beam radiotherapy and HDR brachytherapy for cervical cancer with a 3D-printed deformable pelvis phantom. Med Phys 2017; 44: 1445-1455.

3. L'Annunziata M, Baradei M. Handbook of radioactivity analysis. Academic Press 2003.

4. Scofield, JH. Theoretical photoionization cross sections from 1 to $1500 \mathrm{keV}$. Lawrence Livermore National Laboratory Rep. UCRL-51326 1973.

5. Kurudirek M. Effective atomic numbers and electron densities of some human tissues and dosimetric materials for mean energies of various radiation sources relevant to radiotherapy and medical applications. Radiat Phys Chem 2014; 102: 139-146

6. Singh VP, Badiger NM. Effective atomic numbers of some tissue substitutes by different methods: A comparative study. J Med Phys 2014; 39: 24-31.

7. Sethi R, Cunha A, Mellis K et al. Clinical applications of custom-made vaginal cylinders constructed using three-dimensional printing technology. J Contemp Brachytherapy 2016; 8: 208-214.

8. Lindegaard JC, Madsen ML, Traberg A et al. Individualised 3D printed vaginal template for MRI guided brachytherapy in locally advanced cervical cancer. Radiother Oncol 2016; 118: 173-175.

9. Van der Walt M, Crabtree T, Albantow C. PLA as a suitable 3D printing thermoplastic for use in external beam radiotherapy. Australas Phys Eng Sci Med 2019; 42: 1165-1176.

10. Dancewicz OL, Sylvander SR, Markwell TS et al. Radiological properties of $3 \mathrm{D}$ printed materials in kilovoltage and megavoltage photon beams. Phys Med 2017; 28: 111-118. 
11. Ricotti R, Vavassori A, Bazani A et al. 3D-printed applicators for high dose rate brachytherapy: Dosimetric assessment at different infill percentage. Phys Med 2016; 32: 1698-1706.

12. Taylor ML, Smith RL, Dossing F, Franich RD. Robust calculation of effective atomic numbers: The Auto-Zeff software. Med Phys 2012; 39: 1769-1778.

13. Sukhikh E, Sukhikh L, Malikov E. Polimer Gafchromic EBT3 films in clinical dosimetry. AMR 2015; 1084: 572-576.

14. Massillon JL, Chiu-Tsao ST, Domingo-Munoz I, Chan MF. Energy dependence of new Gafchromic EBT3 film: dose response curves for $50 \mathrm{kV}, 6$ and $15 \mathrm{MVX}$-ray beams. IJMPCERO 2012; 1: 60-65.

15. Oare C, Wilke C, Ehler E et al. Dose calibration of Gafchromic EBT3 film for Ir-192 brachytherapy source using 3D-printed PLA and ABS plastics. 3D Print Med 2019; 5: 3.

16. Sarfehnia A, Kawrakow I, Seuntjens J. Direct measurement of absorbed dose to water in HDR 192Ir brachytherapy: water calorimetry, ionization chamber, Gafchromic film, and TG-43. Med Phys 2010; 37: 1924-1932.

17. DeWerd L, Ibbott G, Meigooni A et al. A dosimetric uncertainty analysis for photon-emitting brachytherapy sources: report of AAPM Task Group No. 138 and GEC-ESTRO. Med Phys 2011; 38: 782-801. 